\title{
Recognizing Human Iris by Modified Empirical Mode Decomposition
}

\author{
Jen-Chun Lee ${ }^{1}$, Ping S. Huang ${ }^{2}$, Te-Ming Tu ${ }^{1}$, and Chien-Ping Chang ${ }^{1}$ \\ ${ }^{1}$ Department of Electrical and Electronic Engineering, Institute of Technology, National \\ Defense University, Taoyuan, Taiwan, Republic of China \\ ${ }^{2}$ Department of Electronic Engineering, \\ Ming Chuan University, Taoyuan, Taiwan, Republic of China \\ \{i923002, cpchang, tutm\}@yahoo.com.tw
}

\begin{abstract}
With the increasing needs in security systems, iris recognition is reliable as one important solution for biometrics-based identification systems. Empirical Mode Decomposition (EMD), a multi-resolution decomposition technique, is adaptive and appears to be suitable for non-linear, non-stationary data analysis. This paper presents an effective approach for iris recognition using the proposed scheme of Modified Empirical Mode Decomposition (MEMD) to analyze the iris signals locally. Since MEMD is a fully data-driven method without using any pre-determined filter or wavelet function, MEMD is used as a low-pass filter to extract the iris features for iris recognition. To verify the efficacy of the proposed approach, three different similarity measures are evaluated. Experimental results show that those three metrics have achieved promising and similar performance. Therefore, the proposed method demonstrates to be feasible for iris recognition and MEMD is suitable for feature extraction.
\end{abstract}

Keywords: Biometrics, iris recognition, Empirical Mode Decomposition (EMD), multi-resolution decomposition.

\section{Introduction}

Biometrics is inherently a more reliable and capable technique to identity human's authentication by his or her own physiological or behavioral characteristics. The features used for personnel identification by current biometric applications include facial features, fingerprints, iris, palm-prints, retina, handwriting signature, DNA, gait, etc. [1], [2] and the lowest error recognition rate is achieved by iris recognition [3]. With the increasing interests, more and more researchers gave their attention into the field of iris recognition.

Recently, iris recognition approaches can be roughly divided into four categories: phase-based approaches [4], zero-crossing representation [5], texture analysis [6], [7], and intensity variation analysis [8], [9]. Daugman's algorithm [4] adopted the 2D Gabor filters to demodulate phase information of iris. Each phase structure is quantized to one of the four quadrants in the complex plane. The Hamming distance was further used to measure the 2048-bits of iris code. Boles and Boashash [5] 
proposed the zero-crossing of 1D wavelet transform to represent distinct levels of a concentric circle for an iris image, and then two dissimilarity functions were used for matching. Wildes et al. [6] analyzed the iris texture using the Laplacian pyramids to combine features from four different resolutions. Normalized correlation is selected to decide whether the input image and the enrolled image belong to the same class. L. Ma et al. [8], [9] proposed a local intensity variation analysis-based method and adopted the Gaussian-Hermite moments [8] and dyadic wavelet [9] to characterize the iris image for recognition.

Feature extraction is a crucial processing stage for pattern recognition. Traditionally, basis decomposition techniques such as Fourier decomposition or Wavelet decomposition are selected to analyze real world signals [10]. Also, Fourier and Wavelet descriptors have long been used as powerful tools for feature extraction [10], [11], [12]. However, the main drawback of those approaches is that the basis functions are fixed, and do not necessarily match varying nature of signals. The Empirical Mode Decomposition (EMD) was firstly proposed by Huang et al. [13] for analyzing nonlinear and non-stationary time series. Any complicated data set can be decomposed into a finite and often small number of intrinsic mode function (IMF) components representing the data features. Those extracted components can match the signal itself very well. Motivated by that EMD provides a decomposition method to analyze the signal locally and separate the component holding locally the highest frequency from the rest into a separate IMF, in this paper, EMD technique is modified and refined to extract distinguishable features from iris images, called Modified Empirical Mode Decomposition (MEMD). There are two merits for using MEMD to extract features for iris recognition. First, MEMD is a fully data driven method without using any pre-determined filter [8], wavelet function or Fourier-wavelet basis [12]. Second, MEMD can be easily implemented, the matching time is greatly reduced and the achieved recognition rate is better than the method using EMD for feature extraction. Therefore, the proposed MEMD approach is used to extract residual components from iris images as features for recognition.

This paper is organized as follows. Section 2 introduces preprocessing procedures for iris images. Section 3 and Section 4 describe the details of our proposed approach for feature extraction and matching. Experimental results are demonstrated and discussed in Section 5, prior to Conclusions in Section 6.

\section{Iris Image Preprocessing}

To ensure that correct iris features can be easily extracted from the eye image, it is essential to perform preprocessing to eye images. The human iris is an annular portion between the pupil (inner boundary) and the sclera (outer boundary). The image preprocessing procedures to extract the iris from the eye image operate in three steps. The first step is to locate the iris area. Then, the located iris is normalized to a rectangular window with a fixed size in order to achieve the approximate scale invariance. Finally, illumination and contrast problems are eliminated from the normalized image by image enhancement, and the most irrelevant parts (such as eyelid and eyelashes) are removed from the normalized image as much as possible. 


\subsection{Locating the Iris Area}

In an iris recognition system, iris location is an essential step. Herein, we proposed the method for iris location base on the Thales' theorem that the diameter of a circle always subtends a right angle to any point on the circle's circumference. Fig. 1 shows the Thales' theorem is applied to find the inner and outer boundary of iris. The iris location method is not detailed here, because it is not the focus of this paper. We sum up the main points as follows. Firstly, the dilation and erosion basic morphological operators are used in order to obliterate the illumination influence inside the pupil. Then, a point inside the pupil is found using the method of minimum local block mean and the pupil area gives the minimum average gray value in the eye image. Therefore, the target point $P_{0}$ inside the pupil is found and its coordinate can be computed. Secondly, we rely on the target point and cooperate with the specialized boundary detection mask (SBDM) to locate three points $\left(P_{1}, P_{2}, P_{3}\right.$ and $\left.P_{4}, P_{5}, P_{6}\right)$ along the inner and outer iris boundaries, respectively. The SBDM is constructed by $a \times b$ matrix, as shown in Fig. 2. During the processing, each pixel $(x, y)$ in the search range is considered as the center of SBDM and the corresponding edge intensity is calculated by

$$
e=\left|\sum_{i=x-\frac{b-1}{2}}^{x+\frac{b-1}{2}} \sum_{j=y-\frac{a-1}{2}}^{y+\frac{a-1}{2}} f(i, j) * w(i, j)\right|
$$

where $f(i, j)$ represents the pixel value in an image, $w(i, j)$ is a weighting value of the SBDM. During the search range, we can find that the boundary point appears with the largest edge intensity variation values. Finally, we apply Thales' theorem to calculate the circle parameters such as the circle center $\left(P_{p}\right.$ and $\left.P_{i}\right)$ and its radius $\left(R_{p}\right.$ and $\left.R_{i}\right)$.

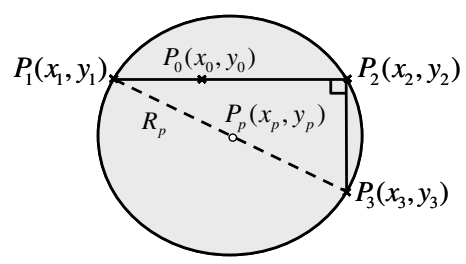

(a)

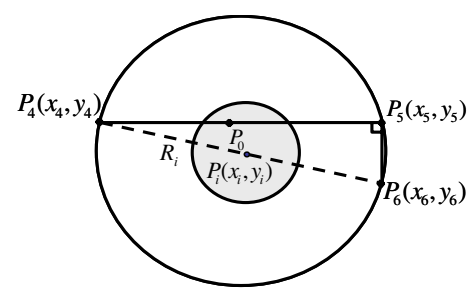

(b)

Fig. 1. Thales' theorem is applied to find (a) the inner and (b) the outer boundary of iris

\begin{tabular}{|l|l|l|l|l|l|l|l|l|l|l|}
\hline 1 & 1 & 1 & 1 & 1 & 0 & -1 & -1 & -1 & -1 & -1 \\
\hline 1 & 1 & 1 & 1 & 1 & 0 & -1 & -1 & -1 & -1 & -1 \\
\hline 1 & 1 & 1 & 1 & 1 & 0 & -1 & -1 & -1 & -1 & -1 \\
\hline
\end{tabular}

Fig. 2. An example of the SBDM $(3 \times 11)$ 


\subsection{Iris Normalization}

The pupil will dilate or constrict when eye images are captured with flash light or under a dark circumstance. For the purpose of achieving more accurate recognition performance, it is necessary to compensate for such deformation. Before using the proposed method for iris recognition, it is required to normalize the iris image, so that the representation is common to all, with similar dimensions.

Normalization process involves in unwrapping the iris and converting it into its equivalent polar coordinates. We transform the circular iris area into a block using Daugman's Rubber sheet model [14]. The pupil center is considered as the reference point and a remapping formula is used to convert the points on the Cartesian scale to the polar scale. In our experiment, the radial resolution and the angular resolution are set to 64 and 512 pixels, respectively.

\subsection{Image Enhancement}

After normalization, iris templates still have low contrast and non-uniform illumination problems. To eliminate the background brightness, the iris template is divided into non-overlapped $16 \times 16$ blocks and their means constitute coarse estimates of background illumination for individual blocks. By using bicubic interpolation, each estimated value is expanded to the size of a $16 \times 16$ block. Then each template block can be enhanced to a uniform light condition by subtracting from the background illumination. After that, the lighting corrected images are enhanced by histogram equalization. It shows clearer texture characteristics of iris than those in Fig. 3(c). Figure 3 illustrates the preprocessing process for the iris image.

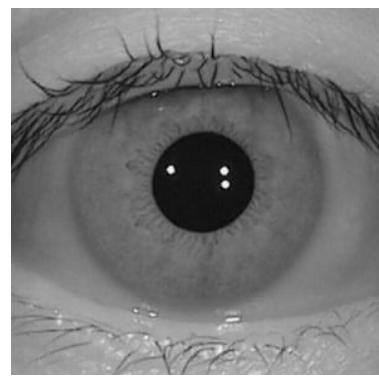

(a)

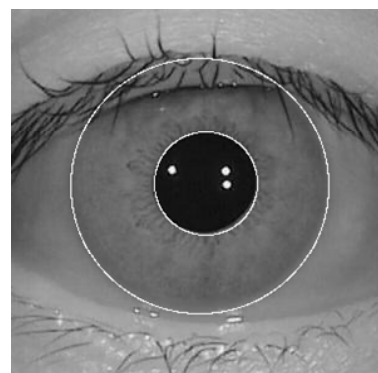

(b)

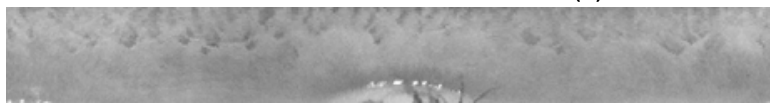

(c)

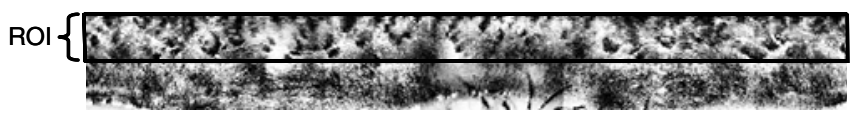

(d)

Fig. 3. Preprocessing of the iris image, (a) the original iris image, (b) the image with located iris area, (c) normalized the iris image, and (d) the region of interesting (ROI) from the enhanced image 


\section{Feature Extraction}

Despite all normalized iris templates have the same size and uniform illumination, there are still eyelashes and eyelids on the templates and those will influence the performance of iris recognition. Therefore, the region of interest (ROI) is selected to remove the influence of eyelashes and eyelids that are shown in Fig. 3(d). The features are extracted only from the upper half region $(32 \times 512)$ which is closer to the pupil and provides the most discriminating information [15].

\subsection{Empirical Mode Decomposition}

Huang et al. [13] introduces a multi-resolution decomposition technique, Empirical Mode Decomposition (EMD) that is adaptive and appears to be suitable for non-linear and non-stationary signal processing method. The major advantage of EMD is that the basis functions are derived directly from the signal itself. Its principle is to decompose adaptively a given signal into components called intrinsic mode functions (IMFs). An IMF is characterized by some specific properties. One is that the number of zero crossings and the number of extrema points are equal or different only by one. Another property of the IMFs is that the mean of its upper and lower envelopes must equal zero. Hence, for a given signal $X$, EMD ends up with a representation of the form:

$$
X=\sum_{i=1}^{n} h_{i}+r,
$$

where $h_{i}$ is the $i$ th mode (or IMF) of the signal, and $r$ is the residual trend (a loworder polynomial component). The sifting procedure generates a finite (and limited) number of IMFs that are nearly orthogonal to each other [13].

\subsection{Modified Empirical Mode Decomposition}

Huang's solution is to find a mean envelope by using cubic spline interpolation through the respective local extrema. It can be argued that repeated iterations using cubic splines in EMD cause the loss of amplitude and frequency information. In this paper, the technique of Modified Empirical Mode Decomposition (MEMD) is proposed to improve EMD for iris feature extraction. The local mean of a signal is accomplished by progressively smoothing the signal using moving averaging. By considering the sample portion of iris data shown in Fig. 4, the local mean involves calculating the mean of the maximum and minimum points of half-wave oscillation of the signal. So the $i$ th mean value $m_{i}$ of each two successive extrema $n_{i}$ and $n_{i+1}$ is given by

$$
m_{i}=\frac{n_{i}+n_{i+1}}{2}
$$

The local mean function is then repeatedly smoothed using this length of moving average until no two successive points with the same value. The smoothing process is shown in Fig. 5. 


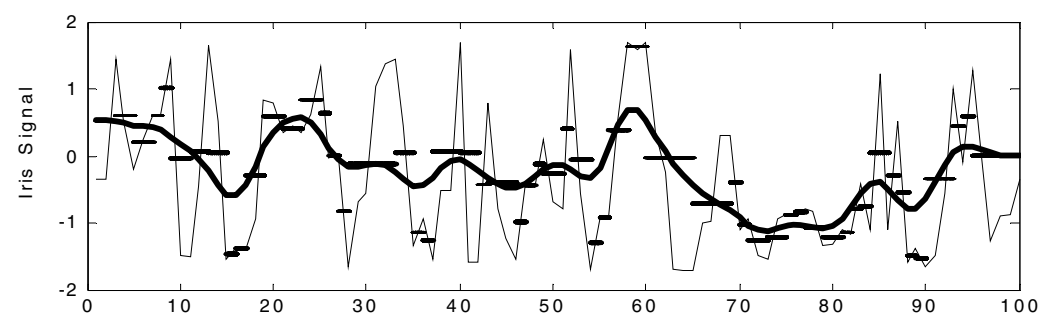

Fig. 4. Sample portion of iris data is displayed as the black line. The local means are shown by horizontal lines computed from the mean of successive extrema. The smoothed local mean is calculated by moving averaging and shown in blod line.

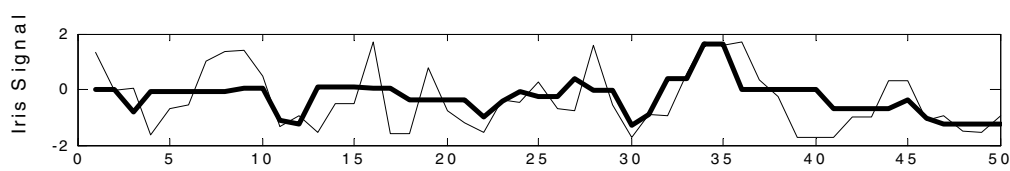

(a)

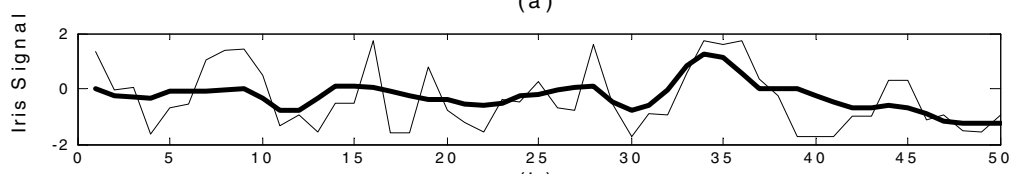

(b)

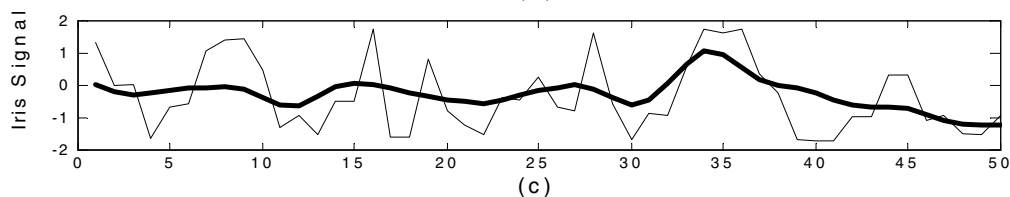

(c)

Fig. 5. The smoothing process ((a)-(c)) of the local mean function using successive applications of a moving average, (c) the final smoothed local mean function

The MEMD principle is similar to EMD that a signal is decomposed into a sum of intrinsic mode functions (IMFs). Also, it has to satisfy two conditions as same as the EMD. Specifically, the first condition is similar to the narrow-band requirement, whereas the second condition modifies a global requirement to a local one by using the local mean defined by the local maxima points and the local minima points, and is necessary to certify that the instantaneous frequency will not have unnecessary fluctuations as induced by asymmetric waveforms. To make use of MEMD for practical applications, the signal must have at least two extrema-one maximum and one minimum to be successfully decomposed into individual IMFs. These IMF components are obtained from the signal by means of an algorithm called sifting process. This algorithm extracts locally for each mode the highest frequency oscillations out of the original signal.

Given those two definitive requirements for an IMF, the sifting process to extract IMFs from a given signal $z(t), t=1, \ldots, T$ is described as follows. 
1) Identify all the maxima and minima in $z(t)$.

2) Calculate the local mean of each two successive extrema using formula (3).

3) The local means are smoothed using moving averaging from a smoothly varying continuous local mean function $m(t)$.

4) Extract the details by $d(t)=z(t)-m(t)$.

5) Check the properties of $d(t)$ :

- If the above-defined two conditions are met, an IMF is derived and $z(t)$ is replaced with the residual $r(t)=z(t)-d(t)$;

- If $r(t)$ is not an IMF, then replace $z(t)$ with $d(t)$.

6) Repeat Steps 1)-5) until the residual satisfies pre-defined stopping criteria.

At the end of this process, the original signal $z(t)$ can then be reconstructed, using the following equation

$$
z(t)=\sum_{i=1}^{n} c_{i}(t)+r_{n}(t),
$$

where $n$ is the number of IMFs, $r_{n}(t)$ denotes the final residue which can be interpreted as the dc component of the signal, and $c_{i}(t)$ are nearly orthogonal to each other, and all have nearly zero means.

In fact, after a certain number of iterations, the produced signals do not carry significant physical information. To avoid this situation, we can stop the sifting process by limiting the normalized standard deviation (SD), computed from two consecutive sifting results. The SD is defined as

$$
\mathrm{SD}=\sum_{t=1}^{T} \frac{\left|z_{j}(t)-z_{j+1}(t)\right|^{2}}{z_{j}^{2}(t)},
$$

the SD is usually set between 0.2 and 0.3 .

As the decomposition process proceeds, the time scale increases, and hence, the mean frequency of the mode decreases. Based on this observation, we may devise a general purpose time-space filtering as

$$
z_{l h}(t)=\sum_{i=1}^{h} c_{i}(t),
$$

where $l, h \in[1, \ldots, n], l \leq h$. For example, when $l=1$ and $h<n$, it is a high-pass filtered signal; when $l>1$ and $h=n$, it is a low-pass filtered signal; when $1<l \leq h<n$, it is a band-pass filtered signal. The above equation forms the basis to our application of iris data described below, where we use it as a low-pass filtering.

To associate with iris recognition, we also present the results of MEMD decomposition for iris images in Fig. 6. Note that the ROI of the normalized iris image is converted into a 1-D feature sequence by concatenating its rows. For the purposes of easy comparison, Figure 6 shows only the first 500 components from their original feature sequences. To demonstrate the similarity of two iris images from the same person captured at different time, it is easily proved by checking those corresponding circles marked in Fig. 6(a) and 6(b). Also, those circles marked in Fig. 6(a) and 6(c) point out the differences of two iris images from two different persons. 


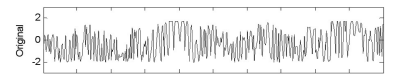

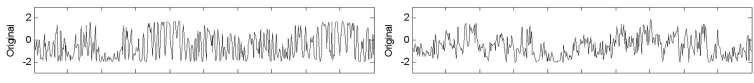

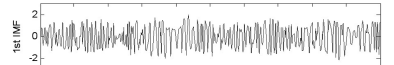

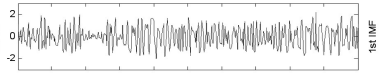

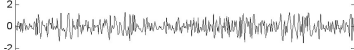

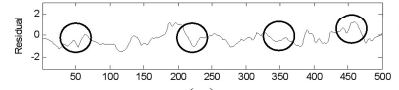

(a)

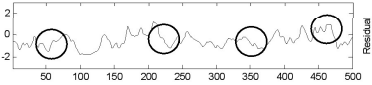

(b)

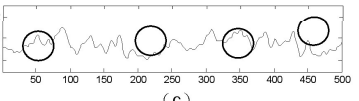

Fig. 6. (a) and (b) show the MEMD decomposition results of two iris images from the same person. (a) and (c) show the MEMD results of two iris images from two different persons.

\subsection{Feature Vector}

For the ROI of each normalized iris image $I$, pixel sequences from adjacent rows are concatenated to form the 1-D vector $V$ represented by

$$
V=\left\{I_{1} \cdots I_{x} \cdots I_{K}\right\}=\left\{v_{1}, v_{2}, \cdots v_{j}, \cdots, v_{n}\right\},
$$

where $I_{x}$ denotes gray values of the $x$ th row in the image $I, v_{j}$ defines the pixel value of position $j$ inside the vector $V$, and $n$ is the number of total components, herein, $n=32 \times 512=18634$. After concatenation and before applying MEMD, linear re-scaling [16] is applied to each vector to adjust the average of each data set to zero and to normalize the standard deviation to unity before further using the ROI vector. After MEMD calculation, the feature vector of each MEMD residual from the 1-D vector can be obtained by

$$
\mathbf{R}^{m}=\left\{R_{1}^{m}, R_{2}^{m}, \cdots R_{j}^{m}, \cdots, R_{n}^{m}\right\},
$$

where $\mathbf{R}^{m}$ represents the $m$ th residual result from MEMD and $R_{j}^{m}$ denotes the feature value from the $j$ th position of the $\mathbf{R}^{m}$.

\section{Iris Matching}

A suitable similarity measure is essential for precise matching between feature vectors. In this article, three different similarity measures used as the matching criterion are:

1) The mean of the Euclidean distances (MED) measure:

$$
d_{1}(p, q)=\sqrt{\frac{1}{M} \sum_{i=1}^{M}\left(p_{i}-q_{i}\right)^{2}},
$$

where $M=K \times L$ is the dimension of the feature vector, $p_{i}$ is the $i$ th component of sample feature vector, and $q_{i}$ is the $i$ th component of unknown sample feature vector. 
2) The cosine similarity measure:

$$
d_{2}(p, q)=1-\frac{p}{\|p\|} \cdot \frac{q}{\|q\|},
$$

where $p$ and $q$ are two different feature vector. $\|\bullet\|$ indicates the Euclidean norm. The range of $\frac{p}{\|p\|} \bullet \frac{q}{\|q\|}$ is $[0,1]$. The more similar the two vectors are, the smaller the $d_{2}(p, q)$ value is.

3) The binary Hamming distance (HD) measure:

$$
d_{3}(p, q)=\frac{1}{M} \sum_{i=1}^{M} p_{i} \oplus q_{i}
$$

where $\oplus$ denotes Exclusive-OR, $M$ is the length of the binary sequence. $p_{i}$ is the $i$ th component of the database sample feature vector, and $q_{i}$ is the $i$ th component of the unknown sample feature vector.

\section{Experimental Results}

To evaluate the performance of the proposed approach at iris recognition, varied experiments are conducted in this section. In the verification mode, the ROC curve that depicts the relationship of false acceptance rate (FAR) versus false reject rate (FRR) is used. Hence, ROC curve is normally used to measure the accuracy of matching process showing the achieved performance of an algorithm. Meanwhile, the equal error rate (EER) is also used for performance evaluation. In the recognition mode, the correct recognition rate (CRR) is adopted to assess the efficacy of the algorithm.

\subsection{Iris Database}

In our experiments, the test data set is from the CASIA Iris Database [17]. Each image has the resolution of $320 \times 280$ in 8 -bit gray level. This database includes 1,992 iris images from 249 different eyes (hence, 249 different classes) with 8 each. The images are acquired during different sessions and the time interval between two collections is at least one month. In our experiments, three images from each class are randomly selected to constitute the training set, so the entire training set has 747 images. The other five images of each class are used as the test set with the number of 1,245 images. Using those 1,992 different iris images from the CASIA Iris Database, the experiments conducted below are running on the computing environment of $1.8 \mathrm{GHz}$ PC with 736MB RAM using Matlab 6.5.

\subsection{Recognition Results}

Table 1 demonstrates promising recognition results achieved by our proposed MEMD method using three similarity measures from (8)-(10). Note that performance 
differences are not very significant while different similarity measures are used. Only a slightly higher recognition rate of $99.31 \%$ is accomplished by using the MED similarity measure in the identification tests. The verification results are also shown in Figure 7. The achieved Az value (the area under the ROC curve) is up to 0.9927 by the MED similarity measure. Therefore, experimental results show that the proposed iris representation is effective for recognition and the MEMD approach can really extract the promising feature from each iris image.

Table 1. Recognition rates of MEMD by different measures

\begin{tabular}{cc}
\hline Similarity measure & Correct recognition rate (CRR) $\%$ \\
\hline MED & $99.31 \%$ \\
\hline Cosine & $98.78 \%$ \\
\hline HD & $98.32 \%$ \\
\hline
\end{tabular}

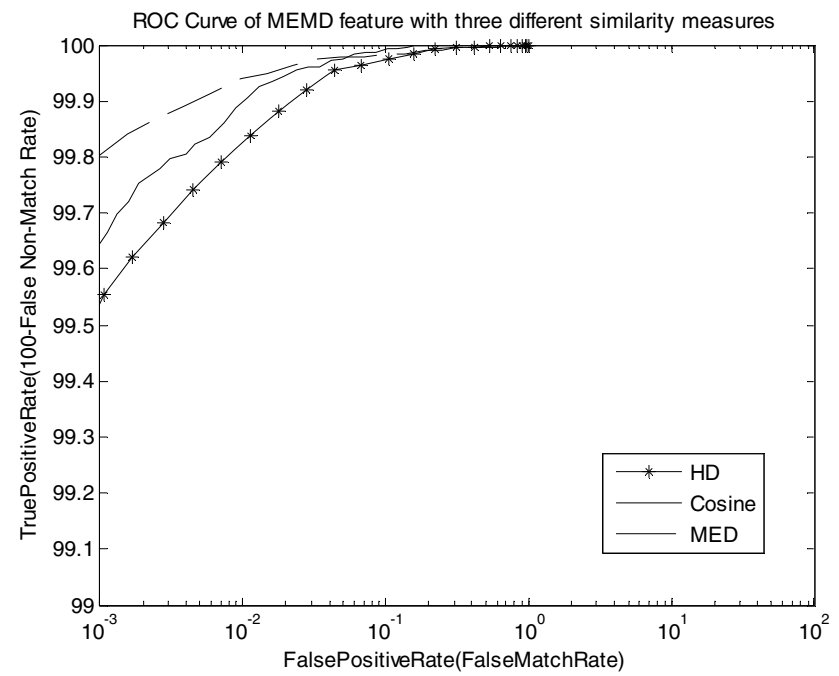

Fig. 7. The ROC curve of MEMD method with different similarity measures

\subsection{Comparison Between MEMD and EMD}

This paper presents the proposed MEMD method and illustrates its performance in iris recognition. MEMD can separate the iris signal into a small set of components and each one could be associated with some aspects of cognitive function.

Although using MEMD on sample test signals produces similar results to those generated by EMD, significant differences between these two schemes should be noted. The MEMD iteration process using smoothed local means appears to be a gentler way of decomposing the data than the cubic spline approach used by EMD. This can be seen in Fig. 8, which compares the intrinsic mode functions calculated by MEMD with the equivalent EMD IMFs. 
Experimental results demonstrated in Section 5.2 reveal that the proposed MEMD technique is an effective scheme for feature extraction from iris images and the MED similarity measure can achieve a correct recognition rate up to $99.31 \%$. Here, we also use the EMD method to extract the iris feature for iris recognition in order to compare with the MEMD method. For the results shown in Table 2 and Fig. 9, we have also implemented the other iris recognition algorithms, the methods of the Fourier-wavelet feature [12] and the Gaussian-Hermite moments [8]. Together with our proposed scheme, four approaches are tested using the 249 classes of the CASIA Iris Database and the cosine similarity measure. Although a slightly lower recognition rate than the approach of Gaussian-Hermite moments is achieved, the proposed method still can fulfill the demand of high accuracy suitable for very high security environments.
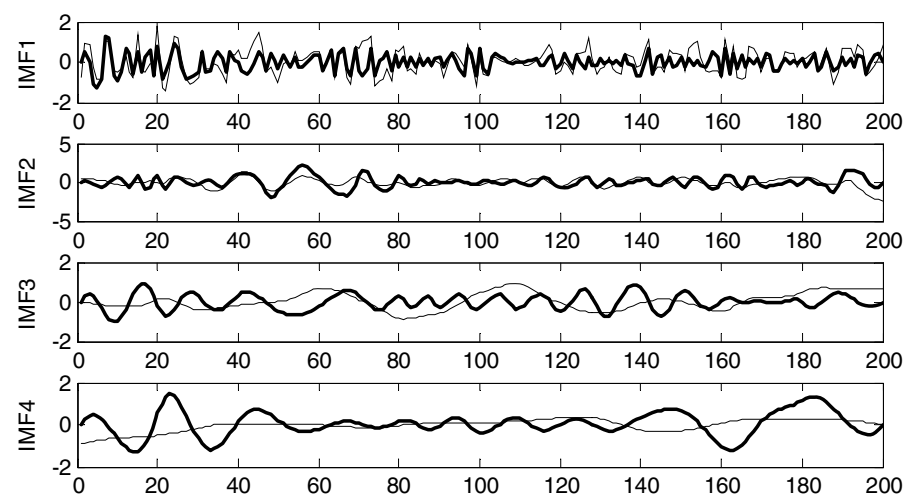

Fig. 8. Comparison between MEMD and EMD intrinsic mode functions. This figure compares four MEMD IMFs (shown in black line) with the four EMD IMFs (shown in bold line) generated from the same iris signal.

Table 2. Typical operating states of the different method

\begin{tabular}{cccc}
\hline Methods & CRR $(\%)$ & $\mathrm{Az}$ & EER (\%) \\
\hline Fourier-wavelet feature[12] & 94.37 & 0.9683 & 5.24 \\
\hline Gaussian-Hermite moments[8] & 99.64 & 0.9989 & 0.29 \\
\hline EMD & 97.22 & 0.9812 & 1.82 \\
\hline Proposed method & 98.78 & 0.9915 & 0.54 \\
\hline
\end{tabular}

To evaluate the computation complexity, Table 3 shows the computational costs consumed by four methods, including the CPU time for feature extraction and matching. In spite of the cost time of EMD for feature extraction is slightly faster than the cost time of MEMD, the computation efficiency of MEMD is still better than the other two methods. Our proposed MEMD method for feature extraction demonstrates to have the desired recognition performance. This can be a potential advantage for iris matching in a large database. 
Based on the previous experimental results and corresponding analysis, we can conclude:

1. The proposed method can achieve high accuracy and fast performance for iris recognition. This indicates that the MEMD technique can extract discriminating features suitable for iris recognition.

2. Although our proposed method presents better computation efficiency than the method of Gaussian-Hermite moments [8], the recognition performance still needs to be improved. Therefore, feature selection is an important research issue in the near future.

Table 3. Comparison of the computational complexity

\begin{tabular}{lccc}
\hline \multicolumn{1}{c}{ Methods } & Feature extraction(s) & Matching(s) & Total times(s) \\
\hline Fourier-wavelet feature[12] & 1.297 & 0.116 & 1.413 \\
\hline Gaussian-Hermite moments[8] & 2.191 & 0.553 & 2.744 \\
\hline EMD & 0.521 & 0.109 & 0.63 \\
\hline Proposed method & 0.985 & 0.108 & 1.093 \\
\hline
\end{tabular}

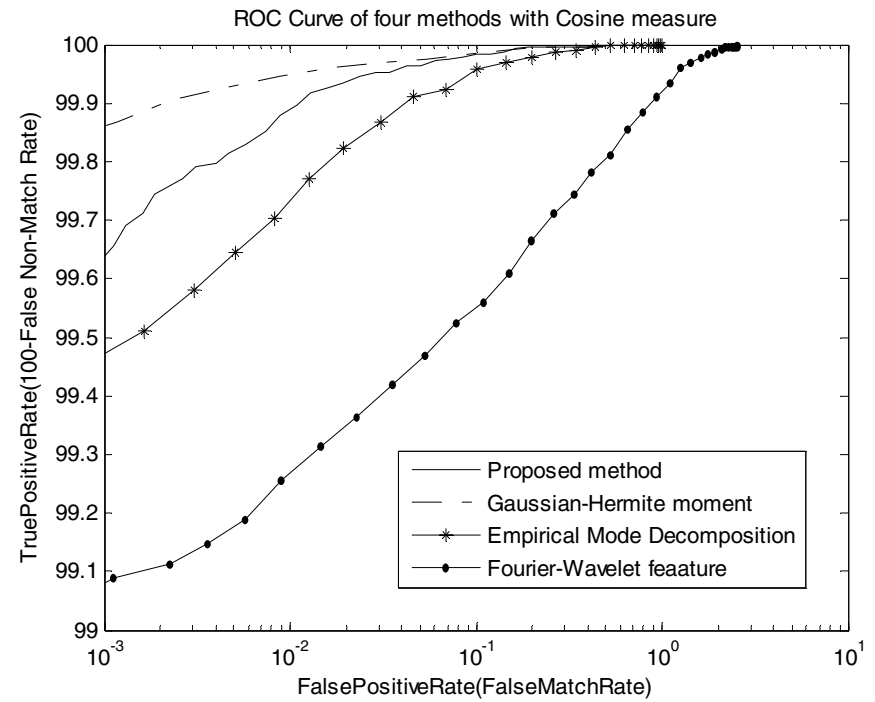

Fig. 9. The ROC curve of different methods using the cosine similarity measure

\section{Conclusions}

In this paper, a novel and effective method of feature extraction for iris recognition is presented, which operates using the Modified Empirical Mode Decomposition (MEMD) technique. The performance of iris recognition achieved by the MEMD approach associated with three different similarity measures has been evaluated. Experimental results have shown that the proposed method can demonstrate eminent 
performance for iris recognition. The best similarity metric is the MED measure and the other two measures also have achieved similar performance more than $98 \%$. Therefore, the proposed method has demonstrated to be promising for iris recognition and MEMD is suitable for feature extraction. In the future, we will ameliorate the template processing method to reduce the influence of light, eyelid, and eyelash. We are also working at increasing the database in order to further verify the performance and trying other possible approaches to improve the classification accuracy.

\section{References}

1. Yanushkevich, S.N.: Synthetic Biometrics: A Survey. In: Proceedings of International Joint Conference on Neural Networks, pp. 676-683 (2006)

2. Miller, B.: Vital Signs of Identity. IEEE Spectrum 31, 22-30 (1994)

3. Mansfield, T., Kelly, G., Chandler, D., Kane, J.: Biometric Product Testing Final Report. issue 1.0, National Physical Laboratory (2001)

4. Daugman, J.: High Confidence Visual Recognition of Persons by a Test of Statistical Independence. IEEE Transactions on Pattern Analysis and Machine Intelligence 15(11), 1148-1161 (1993)

5. Boles, W.W., Boashash, B.: A Human Identification Technique Using Images of the Iris and Wavelet Transform. IEEE Transactions on Signal Processing 46(4), 1185-1188 (1998)

6. Wildes, R., Asmuth, J., Green, G., Hsu, S., Kolczynski, R., Matey, J., McBride, S.: A Machine-Vision System for Iris Recognition. Machine Vision and Applications 9(1), 1-8 (1996)

7. Ma, L., Tan, T., Wang, Y., Zhang, D.: Personal Identification Based on Iris Texture Analysis. IEEE Transactions on Pattern Analysis and Machine Intelligence 25(12), 1519$1533(2003)$

8. Ma, L., Tan, T., Wang, Y., Zhang, D.: Local Intensity Variation Analysis for Iris Recognition. Pattern Recognition 37, 1287-1298 (2004)

9. Ma, L., Tan, T., Wang, Y., Zhang, D.: Efficient Iris Recognition by Characterizing Key Local Variations. IEEE Transactions on Image Processing 13(6), 739-750 (2004)

10. Daubechies, I.: Ten Lectures on Wavelets. SIAM, Philadelphia, Pennsylvania (1992)

11. Wang, S.S., Chen, P.C., Lin, W.G.: Invariant Pattern Recognition by Moment Fourier descriptor. Pattern Recognition 27, 1735-1742 (1994)

12. Huang, P.S., Chiang, C.S., Liang, J.R.: Iris Recognition Using Fourier-Wavelet Features. In: Kanade, T., Jain, A., Ratha, N.K. (eds.) AVBPA 2005. LNCS, vol. 3546, pp. 14-22. Springer, Heidelberg (2005)

13. Huang, N., Shen, Z., Long, S., Wu, M., Shih, H., Zheng, Q., Yen, N., Tung, C., Liu, H.: The Empirical Mode Decomposition and Hilbert Spectrum for Nonlinear and Nonstationary Time Series Analysis. Proc. of the Royal Society of London 454, 903-995 (1998)

14. Daugman, J.: How Iris Recognition Works. IEEE Transactions on Circuits and Systems for Video Technology 14(1), 21-30 (2004)

15. Sung, H., Lim, J.J., Lee, Y.: Iris Recognition Using Collarette Boundary Localization. In: Proceedings of the 17th International Conference on Pattern Recognition, vol. 4, pp. 857860 (2004)

16. Bishop, C.M.: Neural Networks for Pattern Recognition. Oxford University Press, Oxford (1996)

17. Institute of Automation, Chinese Academy of Science, CASIA Iris Image Database, http://www.sinobiometrics.com/chinese/chinese.htm 